\title{
Split-Horizon Scheme for On-line Friction Parameter Estimation
}

\author{
Max Boegli, Tinne De Laet, Joris De Schutter and Jan Swevers \\ Department of Mechanical Engineering, division PMA, K.U.Leuven, 3000 Leuven, Belgium \\ Email: max.boegli@mech.kuleuven.be
}

\begin{abstract}
1 Introduction
Accurate positioning in the nanometer range, e.g. in optical wafer inspection applications in the semiconductor industry, requires active compensation for friction introduced by the roll bearings of the direct drive linear motors that are used to position an XY-table (Fig. 1).

Friction model identification is in most cases carried out in an off-line manner, where separate measurements are performed to identify the presliding and sliding friction characteristics using specific excitation signals. Off-line friction model identification using dynamic non-linear regression are discussed in [1]. The Generalized Maxwell-Slip (GMS) model [2] is known to be able to describe all essential friction characteristics. Moreover, there is a need to update on-line friction parameters during regular point-topoint motions.
\end{abstract}

\section{Estimation Algorithm}

In the GMS model, the friction force is the scalar product of a stiffness vector $k$ times a state vector $z$ plus a viscous term: $F_{f}=k^{T} z+\sigma v$. The state vector $z$ consists of $N$ internal elements, where each single element can either be in a sticking or sliding mode.

Parameter estimation of the GMS model is a non-linear and non-smooth optimization problem due to a switch between stick and slip phases. This non-smooth effect can be reduced by dividing the model parameters into two separate sets and the motion data into zones, each linked to either the presliding or the sliding friction mode (i.e. split horizon). First, friction parameters such as the coulomb force, the attraction parameter and the viscous coefficient are estimated while in sliding mode (all elements are slipping). Then, in a second step, the entry into presliding mode (close to velocity reversal where all or some elements are sticking) permits to fine tune the stiffness coefficients of the internal elements.

Based on available control input $u$, which represents the actuator force, and position $x$, one can estimate friction parameters $p$ such that the estimated friction $\hat{F}_{f}$ minimizes the sum of squared-errors (SSE) between $u$ and the simulated control input $\hat{u}=m \hat{a}+\hat{F}_{f}=h(z(\hat{v}, p), \hat{a})$ assuming a known mass $m$ and good estimates of velocity $\hat{v}$ and acceleration $\hat{a}$. For each zone, slipping and presliding mode, a non-linear LS minimization problem is solved over a finite horizon $K-L$ : $\min _{z_{L}, p} \sum_{k=L}^{K}\left\|u_{k}-h\left(z_{k}\left(v_{k}, p\right), a_{k}\right)\right\|_{2}^{2} \quad$ s.t. $z_{k+1}=g\left(z_{k}, v_{k}, p\right)$.

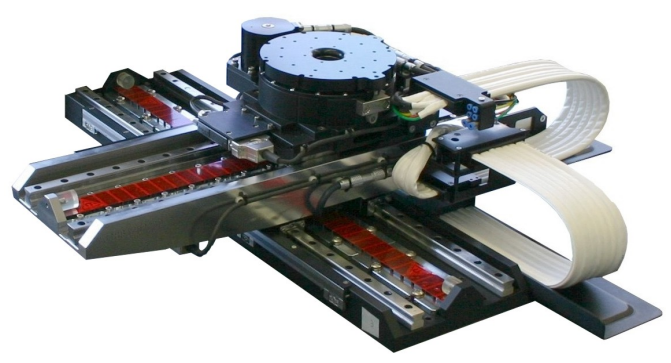

Figure 1: Positioning table for wafer inspection

The optimized variables are the set of parameters $p$ and the initial state vector value $z_{L}$. The state equation model $g($.$) is$ introduced via the equality constraint.

\section{Preliminary results and future work}

Preliminary results on on-line friction parameter estimation for the GMS friction model show an improvement with respect to accuracy and calculation time by applying a split horizon, dividing the motion data and model parameters into separate sets, instead of a single horizon scheme. Further analysis will be carried out to adapt on-line the presliding hysteresis shape of the GMS friction model. Real-time implementation of the constrained non-linear least-squares optimization problem will be implemented with qpOASES, an on-line active set QP solver, and tested on an ETEL XY positioning table (Fig. 1) in a real-time application to compensate friction.

\section{References}

[1] D. D. Rizos and S. D. Fassois, "Friction Identification Based upon the LuGre and Maxwell Slip Models," IEEE Trans. on Control Systems Technology, vol. 17, no. 1, 2009.

[2] F. Al-Bender, V. Lampaert and J. Swevers, "The Generalized Maxwell-Slip Model: A Novel Model for Friction Simulation and Compensation," IEEE Trans. on Automatic Control, vol. 50, no. 11, 2005.

Acknowledgements This work benefits from K.U.Leuven-BOF $\mathrm{PFV} / 10 / 002$ Center-of-Excellence Optimization in Engineering (OPTEC), the Belgian Programme on Interuniversity Attraction Poles, initiated by the Belgian Federal Science Policy Office (DYSCO), and K.U.Leuven's Concerted Research Action GOA/10/11. Tinne De Laet is a Postdoctoral Fellow of the Research Foundation - Flanders (FWO-Vlaanderen). Also, the Swiss Company ETEL is gratefully acknowledged for its support. 